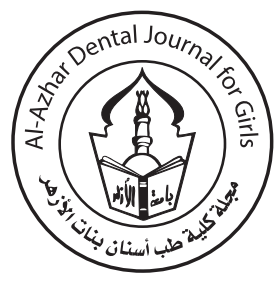

\title{
Electromyographic Evaluation of Jaw Muscles in Edentulous Patients with Implant-Supported Prosthesis
}

\section{Shereen M Kabeel ${ }^{(1)}$ and Dina M Kholief ${ }^{(2)}$}

Codex : 47/1810

azhardentj@azhar.edu.eg

http://adjg.journals.ekb.eg

\section{KEYWORDS}

Mastication,

Implant-supported prostheses,

Electromyography.

\begin{abstract}
The purpose of this study was to evaluate the effect of implant-supported oral rehabilitation in the mandible on the electromyographic (EMG) activity during chewing in completely edentulous individuals. Material and methods: Twelve completely edentulous patients were selected for this study, being six females and six males, aged 55 to 65 years old. The patients were divided into three groups according to the treatment protocol; (GI) All the completely edentulous patients had conventional maxillary and mandibular complete denture, (GII) six patients had two mandibular implants supported overdenture at the canine region and (GIII) six patients had one mandibular implant supported over-denture at the symphesial area. All patients were submitted to EMG evaluation of the masseter, and the temporalis muscles, after three months of placement of the conventional prosthesis and after three months of both implant-supported systems by using different textures of foods. The recordings data were analyzed using One Way ANOVA followed by post hoc analysis using LSD test. $\mathrm{P}<0.05$ was considered statistically significant. Results: the highest EMG activity of the masseter and temporalis muscles was recorded by chewing soft and hard foods in GI, while the reduction of muscular activity after implant supported oral rehabilitation was seen. No significant differences in the analyzed EMG parameters were found between the patients in GII and GIII. Conclusion: mandibular implant-supported prosthesis in edentulous individuals revealed a decrease in EMG amplitude for the masseter and temporalis muscles during chewing, which may indicate adaptation to new conditions of stability provided by support of the complete denture in the mandibular arch.
\end{abstract}

\section{INTRODUCTION}

The aging process causes physiological changes that affect the whole organism. Specifically in relation to the stomatognathic system, in addition to tooth loss, there is reduced masticatory force, alveolar bone decomposition, changes in oral mucosa, and reduction in the number of functional motor units ${ }^{(1)}$.

1. Lecturer of Removable Prosthodontics, Faculty of Dental Medicine For Girls, Al-Azhar University.

2. Assistant Professor of Removable Prosthodontics, Faculty of Dental Medicine For Girls, Al-Azhar University. 
For many years, traditional complete denture designs have been modified to gain additional support and stability from a few retained and suitably prepared natural teeth. Such tooth-supported complete dentures or over-dentures considered as an effective alternative to complete dentures ${ }^{(2)}$. Nowadays, over-denture treatment with the use of implants has become popular for edentulous elderly patients who are maladaptive to complete dentures ${ }^{(3)}$.

Implant-supported prosthesis may be considered a viable and efficient technique for the rehabilitation of partial or full edentulism, and this was confirmed by long-term studies ${ }^{(4,5)}$. A well-planned and performed implant technique provides safe treatments that can significantly improve the neuromuscular function of the masticatory system, and therefore, the quality of life ${ }^{(6,7)}$.

Electromyography (EMG) is a safe, effective and reliable system, widely used to measure the muscular performance of the stomatognathic system in both static and dynamic activities ${ }^{(8)}$.It forms the only tool to assess the muscle activity of the stomatognathic system since its first concerted use in dentistry by Robert moyers in 1949. Over years clinicians and researchers have tried EMG activity to detect the chewing abilities in denture wearers ${ }^{(9)}$.

Observation of the effect of tooth loss in elderly individuals shows that the presence of natural teeth allows a better masticatory performance compared to the use of removable complete dentures with regards to masticatory efficiency, masticatory time, selection of more consistent foods and EMG activity ${ }^{(10)}$. Within this context, edentulous individuals wearing removable complete dentures exhibit reduced muscular activity at the working side, which is poorly adapted to the food texture, as well as decreased EMG activity, especially of the masseter muscle, when compared to dentate individuals ${ }^{(11)}$. The stability of complete dentures also influences the masticatory performance of individuals, as damages to this aspect would result in less regularity and uniformity of mastication cycles ${ }^{(12)}$. Moreover, use of prosthesis damages the sensitivity, oral stereognosis and taste and may also influence the feeding, swallowing and nutritional status of edentulous individuals ${ }^{(13)}$.

The type of dental treatment performed in edentulous individuals also influences the masticatory efficiency, which is greater in individuals rehabilitated with tooth supported and implantsupported dentures compared to removable complete dentures. It should be highlighted that individuals wearing tooth-supported dentures exhibit greater EMG activity of the anterior temporalis muscle during mastication, both in the amplitude and value of integrated activity, compared to individuals wearing removable complete and implant-supported dentures in the mandibular $\operatorname{arch}^{(14)}$. Thus, the use of implant-supported prosthesis leads to improved masticatory function characterized by decrease in EMG activity and reduced masticatory cycle time, although neuromuscular coordination is lower compared to individuals with natural teeth ${ }^{(15,16)}$.

The objective of this study was to evaluate the muscular activity involved in processes of mastication on food of different textures in edentulous individuals using electromyography to compare their performance before and after placement of implant-supported prosthesis in the mandibular arch.

\section{MATERIAL AND METHODS}

\section{Patient selection;}

Twelve completely edentulous patients were selected for this study of both genders (six females and six males), aged 55-65 years old. All subjects were free from systemic diseases as confirmed by history taking and laboratory examinations. All patients were without any noticeable signs and symptoms of stomatognathic system disorder. All selected patients had been wearing dentures for 
about five years and had no abnormal habits such as bruxism, clenching, and tongue thrusting, they did not taking drugs that affect muscle activity, and had adequate mandibular bone for implants insertion. Each patient received a written consent explaining the study description, the advantages and possible risks if present. Patients underwent implant insertion and electromyography recording of masseter and temporalis muscles, in addition to Cone beam computed tomography (CBCT) was made for each patient before surgery for accurate determination of height and width of bone and size of the proposed implant at specific site or sites.

\section{Patient grouping;}

Group I: All the twelve patients received maxillary and mandibular conventional complete dentures, which are in turn divided into two equal groups according to the, position, and number of implant supported over-dentures.

Group II: Six patients received two screw type, root form mandibular implants supported over-denture at the canine region and a complete maxillary removable conventional denture.

Group III: Six patients received one screw type, root form mandibular implant supported over-denture at the symphesial area and a complete maxillary removable conventional denture.

\section{Prosthetic procedures for group I:}

Construction of maxillary and mandibular conventional heat cured acrylic resin complete dentures was done by usual protocol. Final adjustments were made; the dentures were checked for retention and occlusion and patients were asked to wear the denture for three months. Then, EMG recordings were made.

\section{Surgical procedures for group II and III;}

All patients' mandibular dentures were transformed into two or one implant supported mandibular overdenture with ball and socket attachments.

The surgical procedures of implant insertion were done by two-stage technique to minimize the risk of infection. A mucoperiosteal flap was reflected exposing the mandibular inter-foraminal region for optimal implant insertion. The implants (Dentium Superline, Dentium.Co.Ltd,Korea) were derived in position. The implants length was $10 \mathrm{~mm}$ and their diameter was $3.6 \mathrm{~mm}$.All patients received screw shaped, root form implant to permit primary fixation between implant and the bone during initial healing period, also, increasing area of contact between implant surface and surrounding bone, in addition to that, it has been confirmed that the survival of the titanium implants is very high in the anterior region of the mandible with minimal incidence of surgical complications. Thus, the implants in group II were inserted at the canine regions, while in group III, the symphesial region was selected ${ }^{(17)}$.

The pre-planned implants number, type, position, length and width were inserted. The patients were not allowed wearing their dentures for two weeks after surgery then the dentures were relieved at the implant areas to be seated properly in the patient's mouth. Healing period of three months was allowed to assure complete implant bone osseointegration.

Second stage surgery was carried out after three months, attachment installation (Dentium Superline Dentium.Co,Ltd,Korea) and pick up technique was done by auto-polymerized acrylic resin. The finished mandibular implant supported over dentures were inserted into patient's mouth and checked for retention and occlusion, final adjustments were made and the patients were instructed to care and use his or her maxillary complete denture and implant supported mandibular prosthesis for three months (Fig $1 \mathrm{a \& b}$ ). Then, EMG recordings were made. 


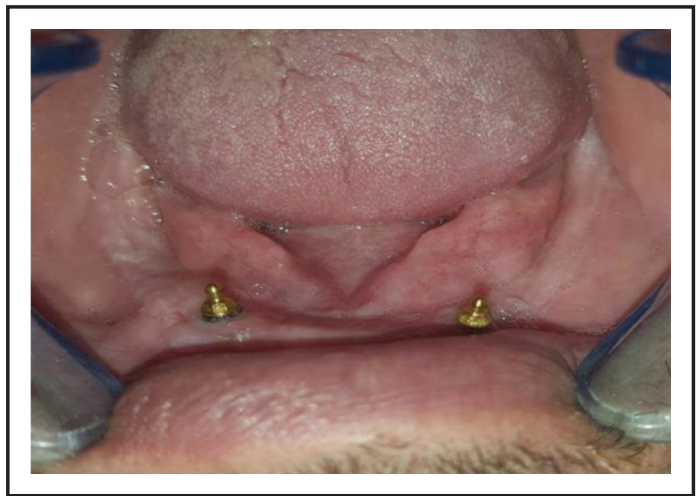

Fig. (1a) Patient has two implants with ball and socket attachment at the canine region

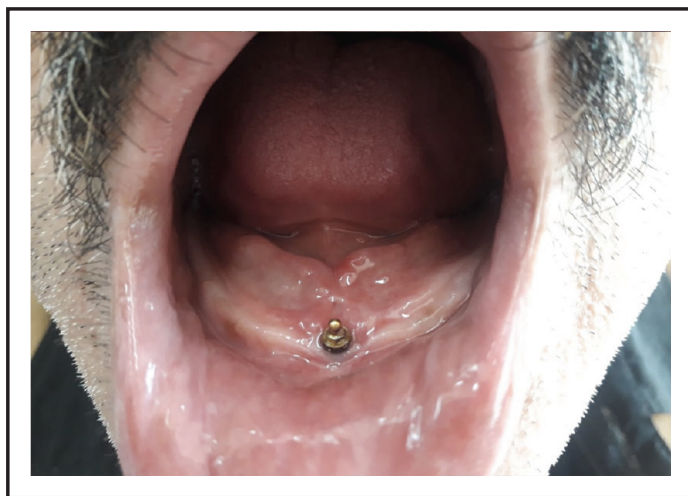

Fig. (1b) Patient has single implant with ball and socket attachment at a symphesial region

\section{Electromyographic activity measurements;}

EMG recording were made after three months for edentulous patients wearing conventional complete denture, then after three months from wearing the two implants supported mandibular over-dentures as well as from wearing the single implant supported mandibular over denture opposed to maxillary conventional complete denture.

EMG recording were made by computer electromyography based data acquisition system (Nemus II) and were expressed as root mean square (RMS). The EMG provided a safe, easy, and non-invasive technique that allowed objective quantification of bioelectric activity of jaw muscles. Standard amounts and sizes of banana and carrots were used to reduce patient variability. These samples of test food were examples of soft and hard food, respectively. During all recordings, the patients were asked to seat with unsupported head and were asked to keep a naturally erect posture.

Before electrode placement, skin was disinfected with ethanol to minimize impedance then electroconductive gel was applied on the electrodes before they contacted the skin and fixed by adhesive tapes. This followed by asking the patients for chewing on his denture or implant supported over-denture teeth at upright position, thus, creating a region of facial muscle contraction which produce impulses. These impulses were printed by laser printer ${ }^{(18)}$.

\section{Statistical Analysis}

Data were collected, revised, coded and entered to the Statistical Package for Social Science (IBM SPSS) version 23. The quantitative data were presented as mean, standard deviations and ranges when their distribution found parametric. The comparison between more than two groups regarding quantitative data with parametric distribution were done by using One-Way ANOVA followed by post hoc analysis using LSD test. The confidence interval was set to $95 \%$ and the margin of error accepted was set to $5 \%$. So, the p-value was considered significant at the level of $<0.05$.

\section{RESULTS}

The success rate for all implant rehabilitation was reported with all patients. All cases finished the study without any complication. All cases of groups (I, II and III) were satisfied with their prosthetic denture and show up a sufficient masticatory efficiency.

The bioelectric activity results during chewing of soft and hard foods, exhibits post prosthetic reduction in EMG amplitude of the masseter muscles in groups II and III, comparing to group I with statistically significant differences ( $\mathrm{p}<0.043$, $\mathrm{p}<0.042$ ) respectively at three months for chewing of soft and hard foods (Table 1, Fig2). 
The bioelectric activity results during chewing of soft and hard foods, exhibits post prosthetic reduction in EMG amplitude of the temporalis muscles in groups II and III, comparing to group I with statistically significant differences $(\mathrm{p}<0.042$, $\mathrm{p}<0.028)$ respectively at three months for chewing of soft and hard foods (Table 1, Fig 2).

Observing the activity of masseter muscle during chewing of soft and hard food, there was decrease in EMG amplitude following implant treatment, though there is no statistically significant difference between groups II and III $(\mathrm{p}<0.442, \mathrm{p}<0.896)$ among the recorded values (Table 1, Fig 2).

Observing the activity of temporalis muscle during chewing of soft and hard food, there was decrease in EMG amplitude following implant treatment, though there is no statistically significant difference between groups II and III $(\mathrm{p}<0.302, \mathrm{p}<0.801)$ among the recorded values (Table 1, Fig 2).

Table (1) Comparison between different numbers of mandibular implants supported over-denture regarding EMG activity of the masseter and temporalis muscles.

\begin{tabular}{|c|c|c|c|c|c|c|c|c|}
\hline \multirow{2}{*}{ Muscle } & \multirow{2}{*}{$\begin{array}{l}\text { Two implant- } \\
\text { supported } \\
\text { Over-denture }\end{array}$} & \multirow{2}{*}{$\begin{array}{l}\text { Single implant- } \\
\text { supported over- } \\
\text { denture }\end{array}$} & \multirow{2}{*}{$\begin{array}{l}\text { Conventi-onal } \\
\text { denture }\end{array}$} & \multicolumn{2}{|c|}{$\begin{array}{c}\text { One Way } \\
\text { ANOVA test }\end{array}$} & \multicolumn{3}{|c|}{ Post hoc analysis } \\
\hline & & & & $\mathbf{F}$ & P-value & $\mathbf{P 1}$ & $\mathbf{P 2}$ & $\mathbf{P 3}$ \\
\hline \multicolumn{9}{|c|}{ Masseter muscle } \\
\hline Soft food & $201.17 \pm 107.73$ & $231.92 \pm 45.87$ & $290.11 \pm 40.58$ & 3.587 & 0.043 & 0.442 & 0.034 & 0.012 \\
\hline Hard food & $256.24 \pm 75.03$ & $261.2 \pm 83.55$ & $335.58 \pm 45.28$ & 3.638 & 0.042 & 0.896 & 0.015 & 0.032 \\
\hline \multicolumn{9}{|c|}{ Temporalis muscle } \\
\hline Soft food & $123.13 \pm 54.73$ & $145.9 \pm 33.17$ & $170.58 \pm 9.21$ & 3.637 & 0.042 & 0.302 & 0.021 & 0.047 \\
\hline Hard food & $183.55 \pm 51.61$ & $188.17 \pm 16.17$ & $229.55 \pm 35.2$ & 4.16 & 0.028 & 0.801 & 0.042 & 0.006 \\
\hline
\end{tabular}

P-value > 0.05: Non significant; P-value $<0.05$ : Significant; $P$-value $<0.01$ : Highly significant

P1: Two implants Vs Single implant P2: Two implant Vs Conventional denture

P3: Single implant Vs Conventional denture

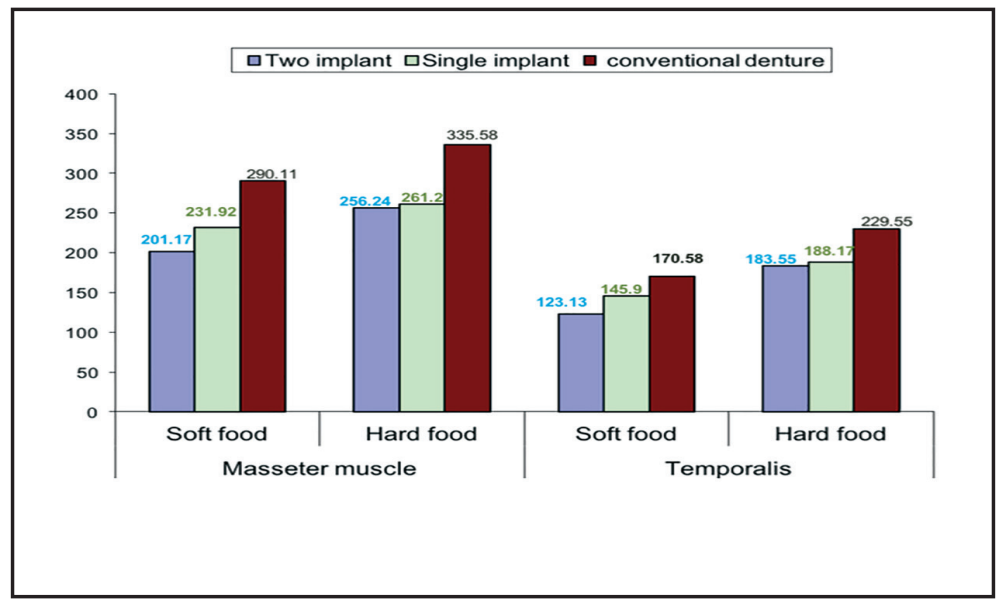

Fig. (2) Bar chart showing Comparison between different numbers of mandibular implants supported over-denture regarding EMG activity of the masseter and temporalis muscles. 


\section{DISCUSSION}

Among the limitations of the study, there was the use of an approved sample: the patients were not randomly selected, and their rehabilitations by prostheses were chosen independently from the present investigations. Only well-satisfied patients were asked to undergo the EMG investigation. Thus, the fulfillment of these results to a wider range of population should be done with caution ${ }^{(19-21)}$.

In the current study, EMG analysis was used to detect and record the muscle fibers activity and to obtain additional information for the diagnosis, management of patients who need oral rehabilitation with implants supported prosthesis and wearing it for three months as a necessary interval for anyone to show satisfactory chewing ability after the restoration was inserted. Also, it should be mentioned that the study subjects chewed a standardized bolus sample that allows focusing on certain parameters ${ }^{(22,23)}$.

EMG has been used to evaluate the masticatory function of implant supported and implant retained overdentures. By using electromyography, the bioelectric activity output of a muscle is proportional to the energy consumed to produce muscle contractions. The evaluation of the masseter and temporalis muscles (right and left sides) is because they are the largest and strongest of the masticatory muscles, the most superficial and are accessible to EMG examination ${ }^{(18)}$

The results of current study, showed significant increased EMG activity of both masseter and temporalis muscles during food chewing in subjects with conventional complete dentures (group I) than the same patients with implant-supported systems (group II and group III), the muscle activity in patients wearing one implant supported prosthesis does not differ from the other group of patients wearing two implants supported over-dentures. This may be explained by complete denture wearers have been demonstrated to perform less organized and uniform chewing cycles because of the poor adaptation to food consistency and the limited stability of the conventional prosthesis, therefore, raising the number of cycles, amplitude and time of chewing ${ }^{(24,25)}$. Also, other explanation for these muscular behaviors could be related to the functional adjustments induced by the implant supported rehabilitation, modulated by the dynamic aging process ${ }^{(26)}$.

The presence of lower numerical values for the masseter and temporalis muscles activity after oral rehabilitation by implant supported prosthesis may be attributed to physiological adjustments expected during the aging process, since studies conducted dentate patients found decrease in EMG amplitude with age ${ }^{(27,28)}$. This population, however, was not investigated in the present study.

In this study, decreasing of muscular activity after implant supported oral rehabilitation of edentulous patients had been observed which is different from the results of other studies that recorded increased muscular activity after implant placement $(29,30)$. Also, other study investigated edentulous patients and observed lower amplitude results for the masseter muscle in patients treated with implant supported prostheses, similar to the findings of the this study ${ }^{(30)}$. However, these contradictory results may be attributed to that; muscle fiber recruitment in individuals rehabilitated via invasive surgical techniques can increase the myoelectric activity to perform the same masticatory patterns of a normal system $^{(31)}$.

Further studies are necessary to observe the behavior patterns of the masticatory muscles in individuals submitted to treatment with implant supported prosthesis, as well as the time of use, adaptation and stability of the prosthesis. Prosthetic rehabilitation is commonly used in dentistry ${ }^{(32)}$. Therefore, research on the topic is of paramount importance to achieve the correct muscle behavior pattern for the proposed treatment. 


\section{CONCLUSION}

Rehabilitation of mandibular implant-supported over-dentures incompletely edentulous patients disclosed a reduction in EMG amplitude for masseter and temporalis muscles during chewing of soft and hard food, which may denote adaptation to new conditions of elite stability and superior support of the complete denture in the mandibular arch.

\section{REFERENCES}

1. Alajbeg IZ, Valentic-Peruzovic M, Alajbeg I, Illes D, Celebic A. The influence of dental status on masticatory muscles activity in elderly patients. Int $\mathbf{J}$ Prosthodont. 2005; 18:333-8.

2. Mericske-Stern R, SteinlinSchaffner T, Marti P, Geering AH. A Peri-implant mucosal aspect of ITI implants supporting overdentures. A five-year longitudinal study. Clin Oral Implants Res1994; 5:9-18.

3. Morais JA, Heydecke G, Pawliuk J, Lund JP, Feine JS. The effects of mandibular two-implant overdentures on nutrition in elderly edentulous individuals. J Dent Res 2003;82:53-8

4. Ekelund JA, Linquist LW, Carlsson GE, Jemt T. Implant treatment in the edentulous mandible: a prospective study on Branemark system implants over more than 20 years. Int J Prosthodont. 2003; 16:602-608.

5. Jemt T, Johansson J. Implant treatment in the edentulous maxillae: a 15-year follow-up study on 76 consecutive patients provided with fixed prostheses. ClinImplant Dent R. 2006; 8:61-69. Bone quality. Int J Oral Maxillofac Implants. $2012 ; 27: 318-28$.

6. De Rossi M, Santos CM, Migliorança R, Regalo SC. All on Four ${ }^{\circledR}$ fixed implant support rehabilitation: a masticatory function study. Clin Implant Dent Relat Res. 2014; 16:594-600.

7. Maló P, de AraújoNobre M, Lopes A, Ferro A, Moss S. Extramaxillary surgical technique: Clinical outcome of 352 patients rehabilitated with 747 zygomatic implants with a follow-up between 6 months and 7 years. Clin Implant Dent Relat Res. 2015; 17:e153-62.

8. Ferrario VF, Tartaglia GM, Maglione M, Simion M, Sforza C. Neuromuscular coordination of masticatory muscles in sub- jects with two types of implant-supported prostheses. Clin Oral Implan Res. 2004; 15:219-225.
9. Bersani E, Regalo SC, Siéssere S, Santos CM, Chimello DT, De Oliveira RH, et al. Implant-supported prosthesis following Brånemark protocol on electromyography of masticatory muscles. J Oral Rehabil. 2011; 38:668-73.

10. Kapur KK, Garret NR. Studies of biologic parameters for denture design. Part II: comparison of masseter muscle activity, masticator performance, and salivatory secretion rates between denture and natural dentition groups. J Prosthet Dent. 1984; 52:408-13.

11. Veyrune JL, Mioche L. Complete denture wears: electromyography of mastication and texture perception whilst eating meat. Eur J Oral Sci. 2000; 108:83-92.

12. Karkazis HC, Kossioni AE. Surface EMG activity of the masseter muscle in denture wearers during chewing of hard and soft food. J Oral Rehabil. 1998; 25:8-14.

13. Ship JA, Duffy V, Jones JA, Langmore S. Geriatric oral health and its impact on eating. J Am Geriatr Soc. 1996; 44(4):456-64.

14. Chen L, Xie Q, Feng H, Lin Y, Li J. The masticatory efficiency of mandibular implant-supported overdentures as compared with toothsupportedoverdentures and complete dentures. J Oral Implantol. 2002; 28:238-43.

15. Bakke M, Holm B, Gotfredsen K. Masticator function and patient satisfaction with implant-supported mandibular overdentures: a prospective 5-year study. Int J Prosthodont. 2002; 15:575-81.

16. Ferrario VF, Tartaglia GM, Maglione M, Simion M, Sforza C. Neuromuscular coordination of masticator muscles in subjects with two types of implant-supported prostheses. Clin Oral Implants Res. 2004; 15:219-25.

17. Abdelhamid AM, Hanno KI, Imam MH.A prospective cross overstudy to evaluate the effect of two different occlusalconcepts on the masseter muscle activity in implant-retained mandibular .Int J Implant Dent. 2015 Dec; 1(1):32.

18. de Rossi M, Palinkas M, de Lima-Lucas B, Santos CM, Semprini M, Oliveira LF, Hallak-Regalo I, Bersani EO, Miglioranca R, Siéssere S, Hallak-RegaloSC.Masticatory muscle activity evaluation by electromyography in subjects with zygomatic implants Med Oral Patol Oral Cir Bucal. 2017 May 1; 22(3):e392-e397.

19. Al-Omiri, M.,Hantash, R.A. \& Al-Wahadni,A. Satisfaction with dental implants: a literature review. Implant Dentistry. 2005;14: 399-406.

20. Flanagan, D. An overview of complete artificial fixed dentition supported by endosseous implants. Artificial Organs.2005;29: 73-81. 
21. Feine, J.S. \& Lund, J.P. Measuring chewing ability in randomized controlled trials with edentulous populations wearing implant prostheses. Journal of Oral Rehabilitation.2006;33: 301-308.

22. Hugger A, Hugger S, Schindler HJ. Surface electromyography of the masticatory muscles for application in dental practice. Current evidence and future developments. Int $\mathrm{J}$ Comput Dent. 2008; 11:81-106.

23. Regalo SC, Santos CM, Vitti M, Regalo CA, de Vasconcelos $\mathrm{PB}$, Mestriner W Jr, et al. Evaluation of molar and incisor bite force in indigenous compared with white population in Brazil. Arch Oral Biol.2008; 53:282-6.

24. Mishellany-Dutour A, Renaud J, Peyron MA, Rimek F, Woda A. Is the goal of mastication reached in young dentatesageddentates and aged denture wearers? Brit J Nutr. 2008; 99:121-128.

25. Veyrune JL, Lassauzay C, Nicolas E, Peyron MA, Woda A. Mastication of model products in complete denture wearers. Arch Oral Biol. 2007; 52:1180-1185.

26. Berretin-Felix G, Nary Filho H, Padovani CR, Trindade Junior AS, Machado WM. Electromyographic evaluation of mastication and swallowing in elderly individuals with mandibular fixed implant-supported prostheses. J Appl Oral Sci. 2008 Mar-Apr; 16(2):116-21.
27. Vaiman M, Evitar E, Segal S. Surface electromyographic studies of swallowing in normal subjects: a review of 440 adults. Report 1. Quantitative data: timing measures. Otolaryngol Head Neck Surg. 2004; 131:548-55.

28. Vaiman M, Evitar E, Segal S. Surface electromyographic studies of swallowing in normal subjects: a review of 440 adults. Report 2. Quantitative data: amplitude measures. Otolaryngol Head Neck Surg. 2004; 131:773-80.

29. Bakke M, Holm B, Gotfredsen K. Masticator function and patient satisfaction with implant-supported mandibular overdentures: a prospective 5-year study. Int J Prosthodont. 2002; 15:575-81.

30. Chen L, Xie Q, Feng H, Lin Y, Li J. The masticatory efficiency of mandibular implant-supported overdentures as compared with toothsupportedoverdentures and complete dentures.J Oral Implantol. 2002; 28:238-43

31. De Rossi M, Santos CM, Migliorança R, Regalo SC. All on Four ${ }^{\circledR}$ fixed implant support rehabilitation: a masticatory function study. Clin Implant Dent Relat Res. 2014; 16:594-600.

32. vanKampen FM, van der Bilt A, Cune MS, FontijnTekamp FA, Bosman F. Masticatory function with implantsupported overdentures. J Dent Res. 2004; 83:708-11. 